\title{
DISTRIBUTIONAL STUDY OF DE FINETTI'S DIVIDEND PROBLEM FOR A GENERAL LÉVY INSURANCE RISK PROCESS
}

\author{
A. E. KYPRIANOU, ${ }^{*}$ The University of Bath \\ Z. PALMOWSKI, ${ }^{* *}$ Utrecht University and Wroctaw University
}

\begin{abstract}
We provide a distributional study of the solution to the classical control problem due to De Finetti (1957), Gerber (1969), Azcue and Muler (2005), and Avram et al. (2007), which concerns the optimal payment of dividends from an insurance risk process prior to ruin. Specifically, we build on recent work in the actuarial literature concerning calculations of the $n$th moment of the net present value of dividends paid out in the optimal strategy as well as the moments of the deficit at ruin and the Laplace transform of the red period. The calculations we present go much further than the existing literature, in that our calculations are valid for a general spectrally negative Lévy process as opposed to the classical Cramér-Lundberg process with exponentially distributed jumps. Moreover, the technique we use appeals principally to excursion theory rather than integro-differential equations and, for the case of the $n$th moment of the net present value of dividends, makes a new link with the distribution of integrated exponential subordinators.
\end{abstract}

Keywords: Reflected Lévy process; passage problem; integrated exponential Lévy process; insurance risk process; ruin

2000 Mathematics Subject Classification: Primary 60K05; 60K15; 91B30

Secondary 60G70; 60J55

\section{Lévy insurance risk processes}

Recall that the Cramér-Lundberg model corresponds to a Lévy process $X^{\mathrm{CL}}=\left\{X_{t}^{\mathrm{CL}}: t \geq 0\right\}$ with characteristic exponent given by

$$
\Psi^{\mathrm{CL}}(\theta)=-\log \int_{\mathbb{R}} \mathrm{e}^{\mathrm{i} \theta x} \mathrm{P}\left(X_{1}^{\mathrm{CL}} \in \mathrm{d} x\right)=-\mathrm{i} c^{\mathrm{CL}} \theta+\lambda^{\mathrm{CL}} \int_{(0, \infty)}\left(1-\mathrm{e}^{-\mathrm{i} \theta x}\right) F(\mathrm{~d} x)
$$

for $\theta \in \mathbb{R}$, such that $\lim _{t \uparrow \infty} X_{t}^{\mathrm{CL}}=\infty$. In other words, $X^{\mathrm{CL}}$ is a compound Poisson process with arrival rate $\lambda^{\mathrm{CL}}>0$ and negative jumps, corresponding to claims, having common distribution function $F$ with finite mean $1 / \mu^{\mathrm{CL}}$ as well as a drift $c^{\mathrm{CL}}>0$, corresponding to a steady income due to premiums, which necessarily satisfies the net profit condition $c^{\mathrm{CL}}-\lambda^{\mathrm{CL}} / \mu^{\mathrm{CL}}>0$. Suppose instead that we work with a general spectrally negative Lévy process, that is, a Lévy process $X=\left\{X_{t}: t \geq 0\right\}$ with Lévy measure $\Pi$ satisfying $\Pi((0, \infty))=0$. At such a degree of generality, the analogue of the condition $c^{\mathrm{CL}}-\lambda^{\mathrm{CL}} / \mu^{\mathrm{CL}}>0$ may be taken to be $\mathrm{E}\left(X_{1}\right)>0$. Such processes have been considered recently by Huzak et al. (2004a), (2004b), Klüppelberg

Received 27 September 2006; revision received 7 March 2007.

* Postal address: Department of Mathematical Sciences, The University of Bath, Claverton Down, Bath BA2 7AY, UK. Email address: a.kyprianou@bath.ac.uk

** Postal address: Mathematical Institute, University of Wrocław, pl. Grunwaldzki 2/4, 50-384 Wrocław, Poland. Email address: zpalma@math.uni.wroc.pl 
et al. (2004), Doney and Kyprianou (2006), Klüppelberg and Kyprianou (2006), and Furrer (1998) in the context of insurance risk models. In this case, the Lévy-Itô decomposition offers an interpretation for large-scale insurance companies as follows. The characteristic exponent may be written in the form

$$
\begin{aligned}
\Psi(\theta)= & -\log \int_{\mathbb{R}} \mathrm{e}^{\mathrm{i} \theta x} \mathrm{P}\left(X_{1} \in \mathrm{d} x\right) \\
= & \left\{\frac{1}{2} \sigma^{2} \theta^{2}\right\}+\left\{-\mathrm{i} \theta c+\int_{(-\infty,-1)}\left(1-\mathrm{e}^{\mathrm{i} \theta x}\right) \Pi(\mathrm{d} x)\right\} \\
& +\left\{\int_{(-1,0)}\left(1-\mathrm{e}^{\mathrm{i} \theta x}+\mathrm{i} \theta x\right) \Pi(\mathrm{d} x)\right\}
\end{aligned}
$$

for $\theta \in \mathbb{R}$ and $\sigma \geq 0$. The Lévy measure necessarily satisfies $\int_{(-\infty, 0)}\left(1 \wedge x^{2}\right) \Pi(\mathrm{d} x)<\infty$, and the requirement that $X$ drift to $\infty$ implies that $c-\int_{(-\infty,-1)}|x| \Pi(\mathrm{d} x)>0$. Note that when $\Pi(-\infty, 0)=\infty$ the process $X$ enjoys a countably infinite number of jumps over each finite time horizon. We may understand the third bracket in (1) as a Lévy process representing a countably infinite number of arbitrarily small claims compensated by a deterministic positive drift (which may be infinite accordingly with the condition $\left.\int_{(-1,0)}|x| \Pi(\mathrm{d} x)=\infty\right)$, corresponding to the accumulation of premiums over an infinite number of contracts. Roughly speaking, the way in which claims occur is such that, in any arbitrarily small period of time $\mathrm{d} t$, a claim of size $|x|$ is made independently with probability $\Pi(\mathrm{d} x) \mathrm{d} t+o(\mathrm{~d} t)$. The insurance company thus counterbalances such claims by ensuring that it collects premiums in such a way that, in any interval $\mathrm{d} t,|x| \Pi(\mathrm{d} x) \mathrm{d} t$ of its income is devoted to the compensation of claims of size $|x|$. We may understand the second bracket in (1) as coming from large claims which occur occasionally and are compensated for by a steady income at rate $c>0$, as in the Cramér-Lundberg model. Here 'large' is taken to mean claims of size 1 or more. Finally, the first bracket in (1) may be seen as a stochastic perturbation of the system of claims and premium income.

Since the first and third brackets correspond to martingales, the company may guarantee that its revenues drift to $\infty$ over an infinite time horizon by assuming that the latter behaviour applies to the compensated process of large claims corresponding to the second bracket in (1).

\section{De Finetti's dividend problem}

An offshoot of the classical ruin problem for the Cramér-Lundberg process was introduced by De Finetti (1957). His intention was to make the study of ruin under the Cramér-Lundberg dynamics more realistic by introducing the possibility that dividends are paid out to shareholders up to the moment of ruin. Furthermore, the payment of dividends should be made in such a way as to optimize the expected net present value of the total income of the shareholders from time 0 until ruin. Mathematically speaking, De Finetti's dividend problem amounts to solving a control problem (which we state in the next paragraph), but within the framework of the general Lévy insurance risk process described in the previous section.

Suppose that $X$ is a general spectrally negative Lévy process (no assumption is made on its long-term behaviour) with probabilities $\left\{\mathrm{P}_{x}: x \in \mathbb{R}\right\}$ such that under $\mathrm{P}_{x}$ we have $X_{0}=x$ with probability 1. (For convenience we shall write $\mathrm{P}_{0}=\mathrm{P}$.) Let $\pi=\left\{L_{t}^{\pi}: t \geq 0\right\}$ be a dividend strategy consisting of a left-continuous, nonnegative, nondecreasing process adapted to the (completed and right-continuous) filtration, $\left\{\mathcal{F}_{t}: t \geq 0\right\}$, of $X$. The quantity $L_{t}^{\pi}$ thus represents the cumulative dividends paid out up to time $t$ by the insurance company whose risk process is modelled by $X$. The controlled risk process when taking account of dividend strategy $\pi$ is thus 
$U^{\pi}=\left\{U_{t}^{\pi}: t \geq 0\right\}$, where $U_{t}^{\pi}=X_{t}-L_{t}^{\pi}$. We write $\sigma^{\pi}=\inf \left\{t>0: U_{t}^{\pi}<0\right\}$ for the time at which ruin occurs when taking account of dividend payments. A dividend strategy is called admissible if at any time before ruin a lump sum dividend payment is smaller than the size of the available reserves; in other words, if $L_{t+}^{\pi}-L_{t}^{\pi} \leq U_{t}^{\pi}$ for $t<\sigma^{\pi}$. Denoting the set of all admissible strategies by $\delta$, the expected value discounted at rate $q>0$ and associated with the dividend policy $\pi \in \delta$ with initial capital $x \geq 0$ is given by

$$
v_{\pi}(x)=\mathrm{E}_{x}\left(\int_{0}^{\sigma^{\pi}} \mathrm{e}^{-q t} \mathrm{~d} L_{t}^{\pi}\right),
$$

where $\mathrm{E}_{x}$ denotes expectation with respect to $\mathrm{P}_{x}$. De Finetti's dividend problem consists of solving the following stochastic control problem: characterize

$$
v^{*}(x):=\sup _{\pi \in \Pi} v_{\pi}(x)
$$

and, if it exists, establish a strategy, $\pi^{*}$, such that $v^{*}(x)=v_{\pi^{*}}(x)$.

This problem was considered by Gerber (1969), who proved that, for the Cramér-Lundberg model, the optimal value function is a result of a band strategy. This result has been reconsidered very recently by Azcue and Muler (2005) for this class of risk processes. Avram et al. (2007) focused on the general spectrally negative case and found sufficient conditions for the optimal strategy to consist of a simpler, barrier, strategy. Thus, we will assume from now on that there exists a constant $a^{*} \in(0, \infty)$ such that the optimal dividend strategy, $\pi^{*}$, corresponds to reducing the risk process $U_{t}^{\pi^{*}}$ to the level $a^{*}$ if $x>a^{*}$ at time $t=0$, by paying the amount $\left(x-a^{*}\right)^{+}$, and then to paying the surplus of the process $X$ above $a^{*}$ as long as it remains above this level. Let $x \leq a^{*}$; otherwise, we let $v^{*}(x)=\left(x-a^{*}\right)+v^{*}(a)$. It is well known that, for $0<x \leq a^{*}$, the corresponding controlled risk process, say $U^{\pi^{*}}$, under $\mathrm{P}_{x}$ is equal in law to the process $a^{*}-Y^{*}=\left\{a^{*}-Y_{t}^{*}: t \geq 0\right\}$ under $\mathrm{P}_{x}$, where

$$
Y_{t}^{*}=\left(a^{*} \vee \bar{X}_{t}\right)-X_{t}
$$

and $\bar{X}_{t}=\sup _{s \leq t} X_{s}$ is the running supremum of the risk process. Moreover, for $x \in\left(0, a^{*}\right)$ we may write

$$
v^{*}(x)=\mathrm{E}_{x}\left(\int_{0}^{\sigma_{a^{*}}} \mathrm{e}^{-q t} \mathrm{~d} L_{t}^{*}\right),
$$

where now $\sigma_{a^{*}}=\inf \left\{t>0: Y_{t}^{*}>a^{*}\right\}$ and $L_{t}^{*}=a^{*} \vee \bar{X}_{t}-a^{*}$.

The optimal strategy associated with De Finetti's dividend problem then yields a net present value of the paid dividends, given by the random variable $\int_{0}^{\sigma_{a^{*}}} \mathrm{e}^{-q t} \mathrm{~d} L_{t}^{*}$. Dickson and Waters (2004) studied distributional aspects of this random variable, in particular its moments, for the special case in which $X$ is a Cramér-Lundberg process and the associated jumps are exponentially distributed. In this paper we build on their results and give a more detailed account of distributional properties of the net present value of the paid dividends in the general case, as well as associated quantities such as the time of ruin, the time at which dividends were last paid, and the time from the latter to ruin. We also analyse the deficit at the ruin time and so-called red period. Dickson and Waters (2004) took an iterative approach to their calculations with the help of integro-differential equations, making heavy use of the fact that jump times form a discrete set and that the jumps themselves are exponentially distributed. Subsequent to submitting this paper, a concurrent paper of Renaud and Zhou (2007) was brought to our attention. In that paper, an iterative approach is applied using the solution to the two-sided exit 
problem independently to prove the same result as appears in our Corollary 1 for the spectrally negative Lévy case. For the Sparre Andersen model, Albrecher et al. (2005) first looked at the moment generating function of the discounted dividend payments and then, under sufficient regularity, rederived the moments from the Taylor expansion. Our approach appeals to Itô's excursion theory and exposes an interesting link with recent work on integrated exponential subordinators. Our method also allows us to derive expressions more general than moments of discounted dividends.

We conclude this section with a brief outline of the remainder of the paper. In the next section we state our two main theorems. In Section 4 we provide a distributional analysis of an integrated bivariate subordinator, which forms a substantial part of the proof of the first of the main results. The proof of the latter is given in Section 5 and the proof of the second main theorem is given in Section 6. We conclude with some further discussion in Section 7.

\section{Main results}

For the sake of clarity we abstract ourselves from De Finetti's dividend problem and suppose that $X=\left\{X_{t}: t \geq 0\right\}$ is any spectrally negative Lévy process under $\mathrm{P}_{x}$, and for a given fixed $a>0$ we write $Y_{t}=\left(a \vee \bar{X}_{t}\right)-X_{t}$. For $t \geq 0$, let $L_{t}=a \vee \bar{X}_{t}-a$ be a version of the Markov local time spent by $Y$ at 0 . By $L_{t}^{-1}$ we denote the inverse local time, defined by

$$
L_{t}^{-1}= \begin{cases}\inf \left\{s>0: L_{s}>t\right\} & \text { if } t<L_{\infty} \\ \infty & \text { otherwise }\end{cases}
$$

\subsection{Distributional identities}

Fundamentally, for $n=0,1,2, \ldots, x \in(0, a]$, and $\lambda, \mu, \kappa \geq 0$, we are interested in the quantity

$$
\Theta(x ; n, \lambda, \mu, \kappa):=\mathrm{E}_{x}\left(\exp \left\{-\lambda L_{L_{\sigma_{a}}-}^{-1}-\mu\left(\sigma_{a}-L_{L_{\sigma_{a}}-}^{-1}\right)-\kappa Y_{\sigma_{a}}\right\}\left(\int_{0}^{\sigma_{a}} \mathrm{e}^{-q t} \mathrm{~d} L_{t}\right)^{n}\right)
$$

where

$$
\sigma_{a}=\inf \left\{t>0: Y_{t}>a\right\}
$$

Note that, in relation to De Finetti's dividend problem, $L$ plays the role of the cumulant dividend payment, $\sigma_{a}$ is the time of ruin of the controlled ruin process, $L_{L_{\sigma_{a}}}^{-1}$ plays the role of the time that dividends were last paid before ruin, and $\sigma_{a}-L_{L_{\sigma_{a}}}^{-1}$ plays the role of time from the last payment of dividends to the time of ruin of the controlled risk process. Finally, $Y_{\sigma_{a}}-a$ is the deficit at the ruin time.

Our first main result is expressed in terms of the scale functions for spectrally negative processes, which we shall briefly introduce. The reader is referred to Chapter 8 of Kyprianou (2006) for a fuller account.

Let

$$
\psi(\theta)=-\Psi(-\mathrm{i} \theta)=\log \mathrm{E}\left(\mathrm{e}^{\theta X_{1}}\right)
$$

be the Laplace exponent of $X$, which is known to be finite for $\theta \in[0, \infty)$ at least. The asymptotic behaviour of $X$ is characterized by $\psi^{\prime}(0+)$ in such a way that $X$ drifts to $\infty$ if $\psi^{\prime}(0+)>0$, drifts to $-\infty$ if $-\psi^{\prime}(0+)>0$ and oscillates if $\psi^{\prime}(0+)=0$. For every $q \geq 0$, there exists a function $W^{(q)}: \mathbb{R} \rightarrow[0, \infty)$ such that $W^{(q)}(x)=0$ for all $x<0$ and is otherwise 
almost everywhere differentiable on $[0, \infty)$, satisfying

$$
\int_{0}^{\infty} \mathrm{e}^{-\lambda x} W^{(q)}(x) \mathrm{d} x=\frac{1}{\psi(\lambda)-q} \text { for } \lambda>\Phi(q),
$$

where $\Phi(q)$ is the largest solution to the equation $\psi(\theta)=q$ (there are at most two). We shall use the shorthand $W^{(0)} \equiv W$ and further assume that $\Pi$, the jump measure of $X$, has no atoms when $X$ has paths of bounded variation, which is a necessary and sufficient condition for $W^{(q)}$ to be continuously differentiable. Associated with $W^{(q)}$ is the function

$$
Z^{(q)}(x):=1+q \int_{-\infty}^{x} W^{(q)}(y) \mathrm{d} y, \quad x \in \mathbb{R}, q \geq 0 .
$$

By a procedure of analytical extension, it is also possible to define $W^{(q)}$ and $Z^{(q)}$ for all $q \in \mathbb{C}$ via the representation

$$
W^{(q)}(x)=\sum_{k \geq 0} q^{k} W^{*(k+1)}(x),
$$

where $W^{* n}(x)$ is the $n$th convolution of $W$; see Chapter 8 of Kyprianou (2006) for further details. (The function $Z^{(q)}$ is defined as above for $q \in \mathbb{C}$.) The functions $W^{(q)}$ and $Z^{(q)}$ are known as scale functions and appear in a number of fundamental identities concerning exit problems; this earns them their title by analogy with the role played by scale functions for one-dimensional diffusions.

There exists a well-known exponential change of measure that one may perform for spectrally negative Lévy processes, namely

$$
\left.\frac{\mathrm{d} \mathrm{P}^{\vartheta}}{\mathrm{dP}}\right|_{\mathcal{F}_{t}}=\mathrm{e}^{\vartheta X_{t}-\psi(\vartheta) t}
$$

for $\vartheta \geq 0$, under which $X$ remains within the class of spectrally negative Lévy processes with parameters changed from those defined via (1) to

$$
\begin{gathered}
\sigma^{\vartheta}=\sigma, \\
c^{\vartheta}=c+\vartheta \sigma^{2}+\int_{(-1,0)} x\left(\mathrm{e}^{\vartheta x}-1\right) \Pi(\mathrm{d} x), \\
\Pi^{\vartheta}(\mathrm{d} x)=\mathrm{e}^{\vartheta x} \Pi(\mathrm{d} x) ;
\end{gathered}
$$

see Proposition 2.1.3 of Kúchler and Sørensen (1997) and Theorem 3.9 of Kyprianou (2006). It turns out that, in the light of this change of measure, it is important to introduce an additional parameter to the scale functions described above. Henceforth, we shall refer to the functions $W_{\vartheta}^{(q)}$ and $Z_{\vartheta}^{(q)}$, where $q \geq 0$ and $\vartheta \geq 0$, as the functions that play the role of the scale functions defined in the previous paragraph when considered under the measure $\mathrm{P}^{\vartheta}$.

We are now ready to state the first of our two main results.

Theorem 1. For $n=1,2,3, \ldots$ and $\lambda, \mu, \kappa \geq 0$, we have

$$
\begin{aligned}
\Theta(x ; n, \lambda, \mu, \kappa)= & n ! \frac{W^{(\lambda+q n)}(x)}{W^{(\lambda+q n)}(a)} \prod_{k=1}^{n} \frac{W^{(\lambda+q k)}(a)}{W^{(\lambda+q k) \prime}(a)} \frac{W^{(\lambda)}(a)}{W^{(\lambda) \prime}(a)} \\
& \times\left\{Z_{\kappa}^{(\mu-\psi(\kappa))}(a) \frac{W_{\kappa}^{(\mu-\psi(\kappa)) \prime}(a)}{W_{\kappa}^{(\mu-\psi(\kappa))}(a)}-(\mu-\psi(\kappa)) W_{\kappa}^{(\mu-\psi(\kappa))}(a)\right\} .
\end{aligned}
$$


Note, in particular, that we have the following corollary, giving a somewhat simpler expression from which the moments of the net present value of paid dividends associated with a barrier strategy can easily be derived.

Corollary 1. For $n=1,2, \ldots$, we have

$$
\mathrm{E}_{x}\left(\left(\int_{0}^{\sigma_{a}} \mathrm{e}^{-q t} \mathrm{~d} L_{t}\right)^{n}\right)=n ! \frac{W^{(q n)}(x)}{W^{(q n)}(a)} \prod_{k=1}^{n} \frac{W^{(q k)}(a)}{W^{(q k) \prime}(a)} .
$$

For the sake of completeness and for later reference, we present a result of Avram et al. (2004).

Lemma 1. For $\mu, \kappa \geq 0$,

$$
\mathrm{E}_{x}\left(\exp \left\{-\mu \sigma_{a}-\kappa\left(Y_{\sigma_{a}}-a\right)\right\}\right)=\mathrm{e}^{\kappa x}\left(Z_{\kappa}^{(v)}(x)-C_{\kappa, \mu}(a) W_{\kappa}^{(v)}(x)\right),
$$

where $v=\mu-\psi(\kappa)$ and

$$
C_{\kappa, \mu}(a)=\frac{v W_{\kappa}^{(v)}(a)+\kappa Z_{\kappa}^{(v)}(a)}{W_{\kappa}^{(v) \prime}(a)+\kappa W_{\kappa}^{(v)}(a)} .
$$

In particular, this lemma gives the joint Laplace transform of the time to ruin and the deficit at ruin.

\subsection{Tail asymptotics}

The second of our main results concerns the case in which the process $X$ satisfies the socalled Spitzer-Doney condition at 0 . This is not compatible with the case in which $X$ is the classical Cramér-Lundberg process. The Spitzer-Doney condition at 0 stipulates that there exists a $\rho \in(0,1)$ such that

$$
\lim _{t \downarrow 0} \frac{1}{t} \int_{0}^{t} \mathrm{P}\left(X_{s} \geq 0\right) \mathrm{d} s=\rho
$$

or, equivalently (see Doney (1995)),

$$
\lim _{t \downarrow 0} \mathrm{P}\left(X_{t} \geq 0\right)=\rho .
$$

A classical example of a spectrally negative Lévy process fulfilling this condition is a spectrally negative, stable process of index $\alpha \in(1,2)$.

Our second main result now follows.

Theorem 2. Suppose that $X$ satisfies the Spitzer-Doney condition at 0 with index $\rho \in(0,1)$. Let $I_{q}=\int_{0}^{\sigma_{a}} \mathrm{e}^{-q t} \mathrm{~d} L_{t}$. Then, for $x \in(0, a]$, as $y \uparrow \infty$,

$$
-\log \mathrm{P}_{x}\left(I_{q}>y\right) \sim(1-\rho) \varphi(y),
$$

where $\varphi(x)$ is the unique solution in $(0, \infty)$ to the equation $\psi(\theta)=\theta x$.

Note, in particular, that the asymptotics above are independent of $q$. Note also that the Spitzer-Doney condition rules out spectrally negative processes of bounded variation. Indeed, it is known (see Bertoin (1996, Theorem VI.3.14, p. 169)) that the Spitzer-Doney condition implies that $\Phi(q)$ is regularly varying at $\infty$ with index $\rho$. However, if $X$ is a spectrally negative Lévy process of bounded variation then it is also known that $\lim _{q \uparrow \infty} \Phi(q) / q=$ $\lim _{\theta \uparrow \infty} \theta / \psi(\theta)=1 / d$, where $d$ is the drift coefficient (which is necessarily strictly positive due to spectral negativity). This implies that $\Phi(q)$ cannot be regularly varying at $\infty$ with index in $(0,1)$ and is thus excluded from the statement of the above theorem. 


\section{Some calculations for an integrated bivariate subordinator}

In order to prove Theorem 1 we first need to move to yet one more degree of abstraction. To motivate why, we must briefly turn to Itô's excursion theory. Denote by $\left\{\left(t, \varepsilon_{t}\right): t \geq 0\right\}$ the Poisson point process of excursions from 0 of $Y$, indexed by the local time at 0 , whose intensity measure necessarily takes the form $\mathrm{d} t \times n(\mathrm{~d} \varepsilon)$ on the product space $[0, \infty) \times \mathcal{E}$, where $\mathcal{E}$ is the space of excursions. The reader is referred to Chapters 5 and 6 of Bertoin (1996) for the necessary background. Assuming that $X_{0}=a$, whence $L_{t}=\bar{X}_{t}$, we may make a change of variables $t \mapsto L_{t}^{-1}$ to deduce that

$$
I_{q}=\int_{0}^{\infty} \mathrm{e}^{-q L_{t}^{-1}} 1_{\left\{\sup _{s \leq t} \bar{\varepsilon}_{s} \leq a\right\}} \mathrm{d} t,
$$

where $\bar{\varepsilon}_{t}$ denotes the height of the excursion at local time $t$ and $1_{\{\cdot\}}$ denotes the indicator function. As a consequence of the fact that excursions form a Poisson point process, we identify the above integral as belonging to the class of integrals of the form

$$
\int_{0}^{\infty} \mathrm{e}^{-q \xi_{t}} 1_{\left\{\sup _{s \leq t} \Delta \eta_{s} \leq a\right\}} \mathrm{d} t
$$

where $\Delta \eta_{t}=\eta_{t}-\eta_{t-}$ and $(\xi, \eta)=\left\{\left(\xi_{t}, \eta_{t}\right): t \geq 0\right\}$ is a bivariate subordinator with respect to some probability measure which we shall denote by $\boldsymbol{P}$ in the sequel.

We thus devote the remainder of this section to a study of results concerning the object in (3) that will be of use later on. Note that integrals such as those in (3) are very close in nature to objects, known as integrated exponential subordinators, which have received some attention recently. See, for example, the survey in Bertoin and Yor (2005). Not surprisingly, some of our calculations are quite close in nature to those presented in the latter article.

Let us henceforth assume that $\Lambda(q)$ is the Laplace exponent of $\xi$. Assume further that $v_{\Lambda}(q)$ is the jump measure of $\eta$ under the change of measure

$$
\left.\frac{\mathrm{d} \boldsymbol{P}^{\Lambda(q)}}{\mathrm{d} \boldsymbol{P}}\right|_{g_{t}}=\mathrm{e}^{\Lambda(q) t-q \xi_{t}},
$$

where $q>0$ and $\left\{g_{t}: t \geq 0\right\}$ is the filtration (satisfying the usual conditions) generated by the process $(\xi, \eta)$.

Theorem 3. For $n=1,2,3, \ldots$ and $q>0$,

$$
\mathrm{E}\left(\left(\int_{0}^{\infty} \mathrm{e}^{-q \xi_{t}} 1_{\left\{\sup _{s \leq t} \Delta \eta_{s} \leq a\right\}} \mathrm{d} t\right)^{n}\right)=n ! \prod_{k=1}^{n} \frac{1}{\Lambda(q k)+v_{\Lambda(q k)}((a, \infty))} .
$$

Proof. We follow an argument for the calculation of the moments of an integrated exponential Lévy process, given in Bertoin and Yor (2005). To this end, define

$$
J_{t}=\int_{t}^{\infty} \mathrm{e}^{-q \xi_{s}} 1_{\left\{\sup _{u \leq s} \Delta \eta_{u} \leq a\right\}} \mathrm{d} s .
$$

Since

$$
\frac{\mathrm{d}}{\mathrm{d} t} J_{t}^{n}=-n J_{t}^{n-1} \mathrm{e}^{-q \xi_{t}} 1_{\left\{\sup _{u \leq t} \Delta \eta_{u} \leq a\right\}},
$$


we obtain

$$
J_{0}^{n}-J_{t}^{n}=n \int_{0}^{t} \mathrm{e}^{-q \xi_{s}} 1_{\left\{\sup _{u \leq s} \Delta \eta_{u} \leq a\right\}} J_{s}^{n-1} \mathrm{~d} s .
$$

Note also that by the stationary and independent increments of $(\xi, \eta)$ we can write

$$
J_{t}=\mathrm{e}^{-q \xi_{t}} 1_{\left\{\sup _{u \leq t} \Delta \eta_{u} \leq a\right\}} J_{0}^{*},
$$

where $J_{0}^{*}$ is independent of $g_{t}$ and has the same distribution as $J_{0}$. In conclusion, if we let

$$
\Psi_{n}=\mathrm{E}\left(J_{0}^{n}\right)
$$

then

$$
\Psi_{n}\left(1-\mathrm{E}\left(\mathrm{e}^{-n q \xi_{t}} 1_{\left\{\sup _{u \leq t} \Delta \eta_{u} \leq a\right\}}\right)\right)=n \Psi_{n-1} \int_{0}^{t} \mathrm{E}\left(\mathrm{e}^{-n q \xi_{s}} 1_{\left\{\sup _{u \leq s} \Delta \eta_{u} \leq a\right\}}\right) \mathrm{d} s .
$$

Now, using the above change of measure, and the fact that the first arrival of a jump of size $a$ for the process $\eta$ occurs after an exponentially distributed period of time, we note that

$$
\begin{aligned}
\mathrm{E}\left(\mathrm{e}^{-q n \xi_{t}} 1_{\left\{\sup _{u \leq t} \Delta \eta_{u} \leq a\right\}}\right) & =\mathrm{e}^{-\Lambda(q n) t} \boldsymbol{P}^{\Lambda(q n)}\left(\sup _{u \leq t} \Delta \eta_{u} \leq a\right) \\
& =\exp \left\{-\left[\Lambda(q n)+v_{\Lambda(q n)}((a, \infty))\right] t\right\} .
\end{aligned}
$$

Plugging the above back into (4), we find the recurrence relation

$$
\Psi_{n}=n \Psi_{n-1} \frac{1}{\Lambda(q n)+v_{\Lambda(q n)}((a, \infty))} .
$$

The proof now concludes in the obvious way.

The following corollary is rather specific but is precisely what is needed later on.

Corollary 2. Suppose that, for $\theta \geq 0, e_{\Lambda}(\theta)$ is an independent exponential random variable with parameter $\Lambda(\theta)$. (In the usual sense we understand this random variable to equal $\infty$ with probability 1 for $\theta=0$ ). Then, for $n=1,2,3, \ldots, q>0$, and $\theta \geq 0$,

$$
\mathrm{E}^{\Lambda(\theta)}\left[\left(\int_{0}^{e_{\Lambda(\theta)}} \mathrm{e}^{-q \xi_{t}} 1_{\left\{\sup _{s \leq t} \Delta \eta_{s} \leq a\right\}}\right)^{n}\right]=n ! \prod_{i=1}^{n} \frac{1}{\Lambda(\theta+q k)+v_{\Lambda(\theta+q k)}((a, \infty))} .
$$

Proof. We again follow arguments similar to those in Bertoin and Yor (2005). Specifically, in the previous result, we replace $\xi_{t}$ by $\xi_{t}^{\prime}=\xi_{t}+\alpha N_{t}$, where $N=\left\{N_{t}: t \geq 0\right\}$ is an independent Poisson process with rate $\Lambda(\theta)$ and $\alpha>0$. Then, taking account of the fact that, under $\boldsymbol{P}^{\Lambda(\theta)}$, $\xi$ is still a subordinator with Laplace exponent $\Lambda(\theta+q)-\Lambda(q)$, from Theorem 3 we have

$$
\begin{aligned}
\mathrm{E}^{\Lambda(\theta)} & {\left[\left(\int_{0}^{\infty} \mathrm{e}^{-q \xi_{t}^{\prime}} 1_{\left\{\sup _{s \leq t} \Delta \eta_{s} \leq a\right\}} \mathrm{d} t\right)^{n}\right] } \\
& =n ! \prod_{k=1}^{n} \frac{1}{\Lambda(\theta+q k)-\Lambda(\theta)+\Lambda(\theta)\left(1-\mathrm{e}^{-\alpha q k}\right)+v^{(k)}((a, \infty))},
\end{aligned}
$$

where $v^{(k)}(\cdot)$ is the jump measure of $\eta$ under $\boldsymbol{P}^{(k)}$, the latter given by the change of measure

$$
\left.\frac{\mathrm{d} \boldsymbol{P}^{(k)}}{\mathrm{d} \boldsymbol{P}^{\Lambda(\theta)}}\right|_{\mathcal{F}_{t}}=\exp \left\{\left[\Lambda(\theta+q k)-\Lambda(\theta)+\Lambda(\theta)\left(1-\mathrm{e}^{-\alpha q k}\right)\right] t-q \xi_{t}^{\prime}\right\} .
$$

Taking limits as $\alpha \uparrow \infty$, the result now follows by monotone convergence. 


\section{Proof of Theorem 1}

The proof of Theorem 1 follows by combining the series of lemmas presented below. As well as making use of the conclusions of the previous section, we shall make use of the following fundamental property associated with the process of excursions from 0 of $Y$, which was earlier denoted $\left\{\left(t, \varepsilon_{t}\right): t \geq 0\right\}$. Note that the excursion $\varepsilon_{t}$, which occurs at local time $t$, is a process in time and hence has a second index referring to the time spent on the excursion. Strictly speaking, $\varepsilon_{t}=\left\{\varepsilon_{t}(s):=X_{L_{t-}^{-1}}-X_{L_{t-}^{-1}+s}: 0<s \leq L_{t}^{-1}-L_{t-}^{-1}\right\}$ for $L_{t}^{-1}-L_{t-}^{-1}>0$. (See Bertoin (1996, Chapter IV) for further discussion.) Accordingly we refer to a generic excursion as $\varepsilon(\cdot)$ or just $\varepsilon$ for short, as appropriate. Define the set $A$ in the space of excursions to be all excursions whose height is greater than $a$, that is $A=\{\varepsilon \in \mathcal{E}: \bar{\varepsilon}>a\}$. Let

$$
T_{a}=\inf \left\{t>0: \varepsilon_{t} \in A\right\} .
$$

Note immediately that $T_{a}=L_{\sigma_{a}}$. The thinning property of Poisson point processes tells us that

(i) $T_{a}$ is a stopping time with respect to the natural filtration of the underlying Poisson point process and is exponentially distributed with parameter $n(\bar{\varepsilon}>a)$, where $\bar{\varepsilon}$ is the maximum of the generic excursion;

(ii) $\varepsilon_{T_{a}}$ is independent of $\left\{\varepsilon_{t}: t<T_{a}\right\}$ and has law given by

$$
\frac{n(\mathrm{~d} \varepsilon ; \bar{\varepsilon}>a)}{n(\bar{\varepsilon}>a)}
$$

(iii) $\left\{\varepsilon_{t}: t<T_{a}\right\}$ is equal in law to a Poisson point process with intensity $\mathrm{d} t \times n(\mathrm{~d} \varepsilon ; \bar{\varepsilon} \leq a)$, stopped at an independent and exponentially distributed time with parameter $n(\bar{\varepsilon}>a)$.

Our sequence of lemmas now follows.

Lemma 2. For $n=1,2,3, \ldots, x \in(0, a)$, and $\lambda, \mu, \kappa \geq 0$,

$$
\Theta(x ; n, \lambda, \mu, \kappa)=\frac{W^{(\lambda+q n)}(x)}{W^{(\lambda+q n)}(a)} \Theta(a ; n, \lambda, \mu, \kappa) .
$$

Proof. Let

$$
\tau_{a}^{+}:=\inf \left\{t>0: X_{t}>a\right\} \quad \text { and } \quad \tau_{0}^{-}:=\inf \left\{t>0: X_{t}<0\right\} .
$$

The random variable in the expectation described by $\Theta(x ; n, \lambda, \mu, \kappa)$ is identically equal to 0 on the event $\left\{\tau_{a}^{+}>\tau_{0}^{-}\right\}$. On the other hand, on the event $\left\{\tau_{a}^{+}<\tau_{0}^{-}\right\}$, factoring out the time spent to reach $a$ in the exponential involving $\lambda$ and $q$, the strong Markov property and spectral negativity of $X$ imply that

$$
\Theta(x ; n, \lambda, \mu, \kappa)=\mathrm{E}\left(\mathrm{e}^{-(\lambda+q n) \tau_{a}^{+}} 1_{\left\{\tau_{a}^{+}<\tau_{0}^{-}\right\}}\right) \Theta(a ; n, \lambda, \mu, \kappa) .
$$

The result now follows from the identity for the two-sided exit problem of a spectrally negative Lévy process; see Theorem 8.1 of Kyprianou (2006).

Lemma 3. For $n=1,2,3, \ldots$ and $\lambda, \mu, \kappa \geq 0$,

$$
\Theta(a ; n, \lambda, \mu, \kappa)=\frac{W^{(\lambda)}(a)}{W^{(\lambda) \prime}(a)} \mathrm{E}_{a}^{\Phi(\lambda)}\left(\left(\int_{0}^{e_{\Phi(\lambda)}} \mathrm{e}^{-q L_{u}^{-1}} 1_{\left\{u<T_{a}\right\}} \mathrm{d} u\right)^{n}\right) n\left(\mathrm{e}^{-\mu \rho_{a}-\kappa \varepsilon\left(\rho_{a}\right)} ; \bar{\varepsilon}>a\right),
$$

where $\rho_{a}=\inf \{s \geq 0: \varepsilon(s)>a\}$. 
Proof. Using the thinning properties of the Poisson point process of excursions we have

$\Theta(a ; n, \lambda, \mu, \kappa)$

$$
\begin{aligned}
& =\mathrm{E}_{a}\left(\exp \left\{-\lambda L_{T_{a}-}^{-1}-\mu\left(\sigma_{a}-L_{T_{a}-}^{-1}\right)-\kappa Y_{\sigma_{a}}\right\}\left(\int_{0}^{L_{T_{a}-}^{-1}} \mathrm{e}^{-q t} \mathrm{~d} L_{t}\right)^{n}\right) \\
& =\mathrm{E}_{a}\left(\mathrm{e}^{-\lambda L_{T_{a}-}^{-1}}\left(\int_{0}^{T_{a}} \mathrm{e}^{-q L_{t}^{-1}} \mathrm{~d} t\right)^{n}\right) \frac{n\left(\mathrm{e}^{-\mu \rho_{a}-\kappa \varepsilon\left(\rho_{a}\right)} ; \bar{\varepsilon}>a\right)}{n(\bar{\varepsilon}>a)} \\
& =\int_{0}^{\infty} n(\bar{\varepsilon}>a) \mathrm{e}^{-n(\bar{\varepsilon}>a) t} \mathrm{E}_{a}\left(\mathrm{e}^{-\lambda S_{t}}\left(\int_{0}^{t} \mathrm{e}^{-q S_{u}} \mathrm{~d} u\right)^{n}\right) \mathrm{d} t \frac{n\left(\mathrm{e}^{-\mu \rho_{a}-\kappa \varepsilon\left(\rho_{a}\right)} ; \bar{\varepsilon}>a\right)}{n(\bar{\varepsilon}>a)}
\end{aligned}
$$

where $S$ is the subordinator with the same drift as $L^{-1}$ but whose jump measure is given by $n(\zeta(\varepsilon) \in d x ; \bar{\varepsilon} \leq a)$ and $\zeta(\varepsilon)$ is the length of the generic excursion $\varepsilon$. Write $p=n_{\Phi(\lambda)}(\bar{\varepsilon}>a)$, where $n_{\Phi(\lambda)}$ is the excursion measure under $\mathrm{P}^{\Phi(\lambda)}$. Continuing we find, with the help of a change of measure and the thinning properties, that

$$
\begin{aligned}
\Theta & (a ; n, \lambda, \mu, \kappa) \\
& =\int_{0}^{\infty} \mathrm{E}_{a}\left(\mathrm{e}^{-\lambda L_{t}^{-1}}\left(\int_{0}^{t} \mathrm{e}^{-q L_{u}^{-1}} \mathrm{~d} u\right)^{n} 1_{\left\{t<T_{a}\right\}}\right) \mathrm{d} t \times n\left(\mathrm{e}^{-\mu \rho_{a}-\kappa \varepsilon\left(\rho_{a}\right)} ; \bar{\varepsilon}>a\right) \\
& =\int_{0}^{\infty} \mathrm{e}^{-\Phi(\lambda) t} \mathrm{E}_{a}^{\Phi(\lambda)}\left(\left(\int_{0}^{t} \mathrm{e}^{-q L_{u}^{-1}} \mathrm{~d} u\right)^{n} 1_{\left\{t<T_{a}\right\}}\right) \mathrm{d} t \times n\left(\mathrm{e}^{-\mu \rho_{a}-\kappa \varepsilon\left(\rho_{a}\right)} ; \bar{\varepsilon}>a\right) \\
& =\int_{0}^{\infty} \mathrm{e}^{-(\Phi(\lambda)+p) t} \mathrm{E}_{a}^{\Phi(\lambda)}\left(\left(\int_{0}^{t} \mathrm{e}^{-q S_{u}} \mathrm{~d} u\right)^{n}\right) \mathrm{d} t \times n\left(\mathrm{e}^{-\mu \rho_{a}-\kappa \varepsilon\left(\rho_{a}\right)} ; \bar{\varepsilon}>a\right) \\
& =\frac{1}{\Phi(\lambda)+p} \mathrm{E}_{a}^{\Phi(\lambda)}\left(\left(\int_{0}^{e_{\Phi(\lambda)} \wedge e_{p}} \mathrm{e}^{-q S_{u}} \mathrm{~d} u\right)^{n}\right) n\left(\mathrm{e}^{-\mu \rho_{a}-\kappa \varepsilon\left(\rho_{a}\right)} ; \bar{\varepsilon}>a\right) \\
& =\frac{1}{\Phi(\lambda)+p} \mathrm{E}_{a}^{\Phi(\lambda)}\left(\left(\int_{0}^{e_{\Phi(\lambda)}} \mathrm{e}^{-q L_{u}^{-1}} 1_{\left\{u<T_{a}\right\}} \mathrm{d} u\right)^{n}\right) n\left(\mathrm{e}^{-\mu \rho_{a}-\kappa \varepsilon\left(\rho_{a}\right)} ; \bar{\varepsilon}>a\right),
\end{aligned}
$$

where in the penultimate equality $e_{\Phi(\lambda)}$ and $e_{p}$ are independent of one another and of $X$.

To complete the proof recall from Lambert (2000) that $n(\bar{\varepsilon}>a)=W^{\prime}(a) / W(a)$ and from Avram et al. (2004) that $W^{(q)}(x)=\mathrm{e}^{\Phi(q) x} W_{\Phi(q)}(x)$. We may now note that

$$
\frac{1}{\Phi(\lambda)+p}=\frac{W_{\Phi(\lambda)}(a)}{W_{\Phi(\lambda)}(a) \Phi(\lambda)+W_{\Phi(\lambda)}^{\prime}(a)}=\frac{W^{(\lambda)}(a)}{W^{(\lambda) \prime}(a)},
$$

thus, concluding the proof.

Lemma 4. For $\mu, \kappa \geq 0$,

$$
n\left(\mathrm{e}^{-\mu \rho_{a}-\kappa \varepsilon\left(\rho_{a}\right)} ; \bar{\varepsilon}>a\right)=Z_{\kappa}^{(\mu-\psi(\kappa))}(a) \frac{W_{\kappa}^{(\mu-\psi(\kappa)) \prime}(a)}{W_{\kappa}^{(\mu-\psi(\kappa))}(a)}-(\mu-\psi(\kappa)) W_{\kappa}^{(\mu-\psi(\kappa))}(a) .
$$

Proof. This has already been established in (the proof of) Theorem 1 of Avram et al. (2004).

Lemma 5. For $n=1,2,3, \ldots$ and $\lambda \geq 0$, we have

$$
\mathrm{E}_{a}^{\Phi(\lambda)}\left(\left(\int_{0}^{e_{\Phi(\lambda)}} \mathrm{e}^{-q L_{t}^{-1}} 1_{\left\{t<T_{a}\right\}} \mathrm{d} t\right)^{n}\right)=n ! \prod_{k=1}^{n} \frac{W^{(\lambda+q k)}(a)}{W^{(\lambda+q k) \prime}(a)} .
$$


Proof. This result follows as a direct consequence of Corollary 2. Relative to that case, here the role of the term $v_{\Lambda(\theta+q k)}((a, \infty))$ is played by

$$
n_{\Phi(\lambda+q k)}(\bar{\varepsilon}>a)=\frac{W_{\Phi(\lambda+q k)}^{\prime}(a)}{W_{\Phi(\lambda+q k)}(a)}=\frac{W^{(\lambda+q k) \prime}(a)}{W^{(\lambda+q k)}(a)}-\Phi(\lambda+q k),
$$

and $\Phi$ is a Laplace exponent of $L_{t}^{-1}$ (see Chapter VII.1 of Bertoin (1996)). A little algebra is necessary on the right-hand side of (5) to complete the proof.

\section{Proof of Theorem 2}

First we should note that the Spitzer-Doney condition implies that $\Phi(q)=q^{\rho} L(q)$, where $L$ is a slowly varying function at $\infty$; see Theorem VI.14 of Bertoin (1996). Following this observation, the proof now mimics very closely the proof of Proposition 2 of Rivero (2003), at the heart of which is an application of Kashara's Tauberian theorem (see Theorem 4.12 of Bingham et al. (1987)). The arguments go though essentially identically, except in our case it is necessary to verify the following asymptotics:

(i) $\lim _{q \uparrow \infty} n_{\Phi(q)}(\bar{\varepsilon}>a)=0$,

(ii) $\lim \sup _{n \uparrow \infty} n \log n / \log \left(1 / m_{n}\right)=1 / \rho$,

(iii) $\lim _{n \uparrow \infty} m_{n}^{1 / n} \Phi(n)=\mathrm{e}^{\rho}$.

Here

$$
m_{n}=\frac{W^{(q n)}(x)}{W^{(q n)}(a)} \prod_{k=1}^{n} \frac{W^{(q k)}(a)}{W^{(q k) \prime}(a)}=\mathrm{e}^{\Phi(q n)(x-a)} \frac{W_{\Phi(q n)}(x)}{W_{\Phi(q n)}(a)} \prod_{k=1}^{n} \frac{1}{\Phi(q k)+n_{\Phi(q k)}(\bar{\varepsilon}>a)} .
$$

Note that in the last equality we have used the facts that $W^{(q)}(x)=\mathrm{e}^{\Phi(q) x} W_{\Phi(q)}(x)$ and $n_{\Phi(q)}(\bar{\varepsilon}>a)=W_{\Phi(q)}^{\prime}(a) / W_{\Phi(q)}(a)$.

Once we prove (i), the proofs of (ii) and (iii) go through as in Rivero (2003). To this end, we recall from Equation (8.15) and Equation (8.3) and the discussion below it in Kyprianou (2006) that, for a fixed $a>0$,

$0 \leq 1-\psi^{\prime}(\Phi(q)) W_{\Phi(q)}(a)=\mathrm{P}_{a}^{\Phi(q)}\left(\tau_{0}^{-}<\infty\right)=\mathrm{E}_{a}\left(\exp \left\{\Phi(q)\left(X_{\tau_{0}^{-}}-a\right)-q \tau_{0}^{-}\right\} 1_{\left\{\tau_{0}^{-}<\infty\right\}}\right)$.

By taking limits as $q \uparrow \infty$, it is now clear that $\psi^{\prime}(\Phi(q)) W_{\Phi(q)}(a) \rightarrow 1$. Next note, with the help of Equation (7.7) in Kyprianou (2006), that we also have

$$
\psi^{\prime}(\Phi(q)) U_{\Phi(q)}((a, \infty))=1-\psi^{\prime}(\Phi(q)) W_{\Phi(q)}(a)
$$

where $U_{\Phi(q)}$ is the potential measure associated with the descending ladder height process of $X$ under the measure $\mathrm{P}^{\Phi(q)}$. It follows from (6) that $U_{\Phi(q)}$ has a density which, for $x>0$, satisfies

$$
\begin{aligned}
\psi^{\prime}(\Phi(q)) W_{\Phi(q)}^{\prime}(x) \mathrm{d} x & =\int_{0}^{\infty} \psi^{\prime}(\Phi(q)) \mathrm{P}^{\Phi(q)}\left(\hat{H}_{t} \in \mathrm{d} x\right) \mathrm{d} t \\
& =\int_{0}^{\infty} \psi^{\prime}(\Phi(q)) \mathrm{e}^{-\Phi(q) x} \mathrm{E}\left(\mathrm{e}^{-q \hat{L}_{t}^{-1}} 1_{\left\{\hat{H}_{t} \in \mathrm{d} x\right\}}\right) \mathrm{d} t
\end{aligned}
$$


where now $\left\{\left(\hat{L}_{t}^{-1}, \hat{H}_{t}\right): t \geq 0\right\}$ is the descending ladder process. Since $\Phi(q)$ is concave (see Section 5.5 of Kyprianou (2006)) and regularly varying with index $\rho$, it follows that $\psi^{\prime}$ is regularly varying with index $\rho^{-1}-1$. From this we deduce that the measure $\psi^{\prime}(\Phi(q)) W_{\Phi(q)}^{\prime}(x) \mathrm{d} x$ converges weakly to the zero measure as $q \uparrow \infty$. Consequently, since $W_{\Phi(q)}^{\prime}(x)$ is known to be continuous in both its arguments (see Section 8.3 of Kyprianou (2006)), we conclude that it also tends to 0 in the limit as $q \uparrow \infty$.

To conclude the proof of (i) and, hence, that of the theorem, simply recall that

$$
n_{\Phi(q)}(\bar{\varepsilon}>a)=\frac{\psi^{\prime}(\Phi(q)) W_{\Phi(q)}^{\prime}(a)}{\psi^{\prime}(\Phi(q)) W_{\Phi(q)}(a)}
$$

and take limits as $q \uparrow \infty$.

\section{Concluding remarks}

We conclude this paper with some remarks on techniques and results pertaining to some special cases considered by other authors. In what follows we shall write $\Theta(x ; n)$ as shorthand for $\Theta(x ; n, 0,0,0)$.

\subsection{Special examples}

There are few examples for which the scale function is known in explicit form (although Rogers (2000) and Surya (2006) have developed methods to numerically invert the Laplace transform in (2)). One of them is the case in which $X$ is a Cramér-Lundberg process with exponentially distributed jumps. In this case, letting $c^{\mathrm{CL}}>0$ be the premium rate, $\lambda^{\mathrm{CL}}>0$ be the rate of arrival of claims, and $\mu^{\mathrm{CL}}$ be the parameter of the exponentially distributed claims, it is known that

$$
W^{(q)}(x)=\frac{1}{c^{\mathrm{CL}}}\left(k_{+}(q) \mathrm{e}^{r_{+}(q) x}-k_{-}(q) \mathrm{e}^{r_{-}(q) x}\right),
$$

where $k_{ \pm}(q)=\left(\mu^{\mathrm{CL}}+r_{ \pm}(q)\right) /\left(r_{+}(q)-r_{-}(q)\right)$ and

$$
r_{ \pm}(q)=\frac{q+\lambda^{\mathrm{CL}}-\mu^{\mathrm{CL}} c^{\mathrm{CL}} \pm \sqrt{\left(q+\lambda^{\mathrm{CL}}-\mu^{\mathrm{CL}} c^{\mathrm{CL}}\right)^{2}+4 c^{\mathrm{CL}} q \mu^{\mathrm{CL}}}}{2 c^{\mathrm{CL}}} .
$$

The expression for $\Theta(x ; n)$ given in Corollary 1 may then be developed and seen to be consistent with the expression given in Equation (2.7) of Dickson and Waters (2004). Note also that in this case the deficit at the time of ruin is exponentially distributed and independent of the time of ruin $\sigma_{a}$, and that

$$
\mathrm{E}_{x}\left(\mathrm{e}^{-\mu \sigma_{a}}\right)=Z^{(\mu)}(x)-C_{0, \mu}(a) W^{(\mu)}(x)
$$

with $W^{(\mu)}$ as given in (7), which agrees with Equation (4.4) of Dickson and Waters (2004).

Lin and Willmot (2000) and Lin et al. (2003) computed objects such as

$$
\mathrm{E}_{x}\left(\mathrm{e}^{-\mu \sigma_{a}}\left(Y_{\sigma_{a}}-a\right)^{n}\right),
$$

where, in particular, combinations of exponentials and mixtures of Erlang distributions are considered for the jump distribution in the classical Cramér-Lundberg model. In principle the current calculations may be connected to theirs, working with any phase-type distribution, $F$, of the claim size in the Cramér-Lundberg model. That is, let

$$
1-F(x)=\alpha \mathrm{e}^{T x} \mathbf{1},
$$


where $\boldsymbol{T}$ is a subintensity matrix of some killed finite-state Markov process, $\boldsymbol{\alpha}$ is its initial probability vector, and $\mathbf{1}$ denotes a column vector of $1 \mathrm{~s}$. Then

$$
W(x)=\frac{1}{\psi^{\prime}(0+)}\left(1-\sum_{j \in \mathcal{I}} A_{j} \mathrm{e}^{\varrho_{j} x}\right),
$$

where $\mathcal{I}$ is the set of all roots $\varrho_{j}$ that have negative real parts (we assume that they are distinct) and solve the Cramér-Lundberg equation

$$
\psi(\varrho)=0,
$$

and

$$
A_{j}=\lim _{s \rightarrow \varrho_{j}} \frac{\psi^{\prime}(0+)\left(\varrho_{j}-s\right)}{\psi(s)}
$$

with $\psi(\theta)=c^{\mathrm{CL}} \theta+\lambda^{\mathrm{CL}}\left(\boldsymbol{\alpha}(\theta \boldsymbol{I}-\boldsymbol{T})^{-1} \boldsymbol{t}-1\right)$, where $\boldsymbol{t}=-\boldsymbol{T} \mathbf{1}$ and $\mathbf{1}$ is the identity matrix; for details, see Lemma 1 of Asmussen et al. (2004) and Equation (13) of Kyprianou and Palmowski (2005). We recall also that $W^{(q)}(x)=\mathrm{e}^{\Phi(q) x} W_{\Phi(q)}(x)$. Using Lemma 1, (9), and straightforward differentiation, it is in principle possible to calculate (8).

Another example is the case of an $\alpha$-stable process with $\alpha \in(1,2)$. In this case we may take $\psi(\theta)=\theta^{\alpha}$. It is known that for this class of processes the positivity parameter equals $\rho=\alpha^{-1}$, and that

$$
W^{(q)}(x)=\alpha x^{\alpha-1} E_{\alpha}^{\prime}\left(q x^{\alpha}\right),
$$

where $E_{\alpha}$ is the Mittag-Leffler function of index $\alpha$ :

$$
E_{\alpha}(x)=\sum_{n=0}^{\infty} \frac{x^{n}}{\Gamma(1+\alpha n)}
$$

for $x \in \mathbb{R}$. Note that such stable processes do not satisfy the condition $\lim _{t \uparrow \infty} X_{t}=\infty$, but instead oscillate. This puts them within the context of Theorem 2 , which now says that

$$
-\log \mathrm{P}_{x}\left(I_{q}>y\right) \sim\left(\frac{\alpha-1}{\alpha}\right) y^{1 /(\alpha-1)} .
$$

as $y \uparrow \infty$.

\subsection{Integro-differential equations}

In the case in which $X$ is the classical Cramér-Lundberg process with linear drift coefficient $c^{\mathrm{CL}}$ and negative jumps arriving at rate $\lambda^{\mathrm{CL}}>0$ and having distribution function $F$, by conditioning on the first jump Dickson and Waters (2004) showed that $\Theta(x ; n)$ solves the integro-differential equation

$$
c^{\mathrm{CL}} \Theta^{\prime}(x ; n)+\lambda^{\mathrm{CL}} \int_{(0, \infty)}\{\Theta(x-y ; n)-\Theta(x ; n)\} F(\mathrm{~d} y)-q n \Theta(x ; n)=0
$$

on $(0, a)$, with boundary condition $\Theta^{\prime}(a-; n)=n \Theta(a ; n-1)$ for $n=1,2,3, \ldots$ (see their Theorem 2.1 to make the connection with the above equation, noting in particular that $\Theta(x ; n)=0$ for $x<0)$. In the language of extended generators, (10) can be written more simply as

$$
(\Gamma-q n) \Theta(x ; n)=0,
$$

where $\Gamma$ is the extended generator of $X$. 
It is now interesting to note from the conclusion of Lemma 1 that, in the general case, up to a constant which depends on $a, q$, and $n$, we have $\Theta(x ; n) \propto W^{(q n)}(x)$. It is known (see Avram et al. (2004)) that $\left\{\mathrm{e}^{-q n t} W^{(q n)}\left(X_{t}\right): t<\tau_{0}^{-}\right\}$is a martingale and, hence, that $W^{(q n)}$ is in the domain of the extended generator and solves the generator equation $(\Gamma-q n) W^{(q n)}(x)=0$ on $(0, \infty)$ (in particular on $(0, a))$. Therefore, under such circumstances it also follows that (11) holds. We also note that from the conclusion of Corollary 1 that

$$
\Theta^{\prime}(a-; n)=n ! \frac{W^{(q n) \prime}(a)}{W^{(q n)}(a)} \prod_{i=1}^{n} \frac{W^{(q k)}(a)}{W^{(q k) \prime}(a)}=n(n-1) ! \prod_{i=1}^{n-1} \frac{W^{(q k)}(a)}{W^{(q k) \prime}(a)}=n \Theta(a ; n-1) .
$$

In fact, Dickson and Waters (2004) considered (11) in the context of the infinitesimal generator $\Gamma$ for a sufficiently smooth function $\Theta(x ; n)$. 'Sufficiently smooth' here means that the aforementioned generator equation is mathematically well defined. Note that the fact that scale functions are not known necessarily to be smooth enough to use in conjunction with the infinitesimal generator of the associated Lévy process implies that the method of Dickson and Waters (2004) cannot be easily implemented for the general case considered here.

\subsection{The case in which $q=0$}

In Section 3 of Dickson and Waters (2004) it was noted that, for $q=0, \int_{0}^{\sigma_{a}} \mathrm{e}^{-q t} \mathrm{~d} L_{t}=L_{\sigma_{a}}$ (that is, the total dividends paid until ruin) is equal in distribution to a mixture of an atom at 0 and an exponential distribution. Again we remark that their method of proof cannot be applied in the general Lévy process setting as it relies heavily on the fact that in the Cramér-Lundberg model the support of the local-time measure $\mathrm{d} L$, when restricted to any finite time horizon, consists of the union of a finite number of closed intervals.

Nonetheless, their result is true even in the more general context considered here. Taking account of whether or not the process $Y$ hits 0 before exceeding level $a$, for $x>0$ we see that

$$
\begin{aligned}
\mathrm{P}_{x}\left(L_{\sigma_{a}} \in \mathrm{d} z\right) & =\delta_{0}(\mathrm{~d} z) \mathrm{P}_{x}\left(\tau_{a}^{+}>\tau_{0}^{-}\right)+\mathrm{P}_{a}\left(L_{\sigma_{a}} \in \mathrm{d} z\right) \mathrm{P}_{x}\left(\tau_{a}^{+}<\tau_{0}^{-}\right) \\
& =\delta_{0}(\mathrm{~d} z)\left(1-\frac{W(x)}{W(a)}\right)+\frac{W(x)}{W(a)} \mathrm{P}_{a}\left(L_{\sigma_{a}} \in \mathrm{d} z\right) \\
& =\delta_{0}(\mathrm{~d} z)\left(1-\frac{W(x)}{W(a)}\right)+\frac{W(x)}{W(a)} n(\bar{\varepsilon}>a) \mathrm{e}^{-n(\bar{\varepsilon}>a) z} \mathrm{~d} z,
\end{aligned}
$$

where the last equality follows by virtue of the fact that, under $\mathrm{P}_{a}, L_{\sigma_{a}}=T_{a}$, which, according to thinning property (i) of Poisson point processes, is exponentially distributed. Finally recall that $n(\bar{\varepsilon}>a)=W^{\prime}(a) / W(a)$; thus, in conclusion we have

$$
\mathrm{P}_{x}\left(L_{\sigma_{a}} \in \mathrm{d} z\right)=\delta_{0}(\mathrm{~d} z)\left(1-\frac{W(x)}{W(a)}\right)+\frac{W(x) W^{\prime}(a)}{W(a)^{2}} \mathrm{e}^{-z W^{\prime}(a) / W(a)} \mathrm{d} z .
$$

\subsection{The red period}

Denoting by $F_{x}$ the distribution of the deficit $Y_{\sigma_{a}}-a$ under $\mathrm{P}_{x}$, we can also find the Laplace transform of so-called red period, $r$, that is, the duration of the first negative surplus (see Dos Reis (1993) and Dickson and Dos Reis (1996)). Indeed for $s \geq 0$, we have

$$
\begin{aligned}
\mathrm{E}_{x}\left(\mathrm{e}^{-s r}\right) & =\int_{0}^{\infty} \mathrm{E}\left(\mathrm{e}^{-s \tau_{y}^{+}}\right) F_{x}(\mathrm{~d} y)=\int_{0}^{\infty} \mathrm{e}^{-\Phi(s) y} F_{x}(\mathrm{~d} y) \\
& =\mathrm{E}_{x}\left(\mathrm{e}^{-\Phi(s)\left(Y_{\sigma_{a}}-a\right)}\right)=\mathrm{e}^{\Phi(s) x}\left(Z_{\Phi(s)}^{(-s)}(x)-C_{\Phi(s), 0}(a) W_{\Phi(s)}^{(-s)}(x)\right),
\end{aligned}
$$


where Theorem 1 of Kyprianou and Palmowski (2005) has been used in the second equality and Lemma 1 has been used in the last equality. The last expression can be developed further using the relations $W_{\Phi(s)}^{(-s)}(x)=\mathrm{e}^{-\Phi(s) x} W(x)$ and $Z_{\Phi(s)}^{(-s)}(x)=1+s \int_{0}^{x} \mathrm{e}^{-\Phi(s) y} W(y) \mathrm{d} y$, the latter of which is a consequence of Lemma 8.4 of Kyprianou (2006). In conclusion,

$$
\mathrm{E}_{x}\left(\mathrm{e}^{-s r}\right)=\mathrm{e}^{\Phi(s) x}+s \int_{0}^{x} \mathrm{e}^{\Phi(s)(x-y)} W(y) \mathrm{d} y-C_{\Phi(s), 0} W(x),
$$

where now we can write

$$
C_{\Phi(s), 0}=\frac{\Phi(s) \mathrm{e}^{\Phi(s) a}+s \Phi(s) \int_{0}^{a} \mathrm{e}^{\Phi(s)(a-y)} W(y) \mathrm{d} y-s W(a)}{W^{\prime}(a)} .
$$

\section{Acknowledgements}

Both authors acknowledge support from the NWO grant number 613.000.310. The second author acknowledges support from KBN 1P03A03128. Also we would like to thank the editorial office and the referee for bringing the work of Renaud and Zhou (2007) to our attention.

\section{References}

Albrecher, H., Claramunt, M. M. and Mármol, M. (2005). On the distribution of dividend payments in a Sparre Andersen model with generalized Erlang $(n)$ interclaim times. Insurance Math. Econom. 37, 324-334.

Asmussen, S., Avram, F. And Pistorius, M. (2004). Russian and American put options under exponential phase-type Lévy models. Stoch. Process. Appl. 109, 79-111.

Avram, F., Kyprianou, A. E. And Pistorius, M. R. (2004). Exit problems for spectrally negative Lévy processes and applications to (Canadized) Russian options. Ann. Appl. Prob. 14, 215-238.

Avram, F., Palmowski, Z. and Pistorius, M. R. (2007). On the optimal dividend problem for a spectrally negative Lévy process. Ann. Appl. Prob. 17, 156-180.

Azcue P. And Muler, N. (2005). Optimal reinsurance and dividend distribution policies in the Cramér-Lundberg model. Math. Finance 15, 261-308.

Bertoin, J. (1996). Lévy Processes. Cambridge University Press.

Bertoin, J. And Yor, M. (2005). Exponential functionals of Lévy processes. Prob. Surveys 2, 191-212.

Bingham, N. H., Goldie, C. M. And Teugels, J. L. (1987). Regular Variation. Cambridge University Press.

De FinetTi, B. (1957). Su un'impostazion alternativa dell teoria collecttiva del rischio. Trans. XVth Internat. Congr. Actuaries 2, 433-443.

Dickson, D. C. M. And Dos ReIs, A. E. (1996). On the distribution of the duration of negative surplus. Scand. Actuarial J. 2, 148-164.

Dickson, D. C. M. AND Waters, H. R. (2004). Some optimal dividends problems. ASTIN Bull. 34, 49-74.

Doney, R. A. (1995). Spitzer's condition and ladder variables in random walks. Prob. Theory Relat. Fields 101, 577-580.

Doney, R. A. and Kyprianou, A. E. (2006). Overshoots and undershoots of Lévy processes. Ann. Appl. Prob. 16, 91-106.

Dos ReIs, A. E. (1993). How long is the surplus below zero? Insurance Math. Econom. 12, 23-38.

Furrer, H. (1998). Risk processes perturbed by $\alpha$-stable Lévy motion. Scand. Actuarial J. 1998, 59-74.

Gerber, H. U. (1969). Entscheidungskriterien für den zusammengesetzten Poisson-Prozess. Schweiz. Verein. Versicherungsmath. Mitt. 69, 185-228.

Huzak, M., Perman, M., ŠIKIĆ, H. AND VondračEK, Z. (2004a). Ruin probabilities and decompositions for general perturbed risk processes. Ann. Appl. Prob. 14, 1378-1397.

HuZak, M., Perman, M., Šikić, H. And VondračeK, Z. (2004b). Ruin probabilities for competing claim processes. J. Appl. Prob. 41, 679-690.

KlüPPElBERG, C. AND KyPRIANOU, A. E. (2006). On extreme ruinous behaviour of Lévy insurance risk processes. $J$. Appl. Prob. 43, 594-598.

Klüppelberg, C., Kyprianou, A. E. And Maller, R. A. (2004). Ruin probabilities and overshoots for general Lévy insurance risk processes. Ann. Appl. Prob. 14, 1766-1801.

KǗchler, U. ANd Sørensen, M. (1997) Exponential Families of Stochastic Processes. Springer, New York.

Kyprianou, A. E. (2006). Introductory Lectures on Fluctuations of Lévy Processes with Applications. Springer, Berlin. 
Kyprianou, A. E. And Palmowski, Z. (2005). A martingale review of some fluctuation theory for spectrally negative Lévy processes. In Séminaire de Probabilités XXXVIII (Lecture Notes Math. 1857), Springer, Berlin, pp. 16-29.

Lambert, A. (2000). Completely asymmetric Lévy processes confined in a finite interval. Ann. Inst. H. Poincaré. Prob. Statist. 36, 251-274.

Lin, X. S. And Willmot, G. E. (2000). The moments of the time of ruin, the surplus before ruin, and the deficit at ruin. Insurance Math. Econom. 27, 19-44.

Lin, X. S., Willmot, G. E. And Drekic, S. (2003). The classical risk model with a constant dividend barrier: analysis of the Gerber-Shiu discounted penalty function. Insurance Math. Econom. 33, 551-566.

Renaud, J. And Zhou, X. (2007). Distribution of the present value of dividend payments in a Lévy risk model. $J$. Appl. Prob. 44, 420-427.

Rivero, V. (2003). A law of iterated logarithm for increasing self-similar Markov processes. Stoch. Stoch. Reports $\mathbf{7 5}$, 443-472.

Rogers, L. C. G. (2000). Evaluating first-passage probabilities for spectrally one-sided Lévy processes. J. Appl. Prob. 37, 1173-1180.

Surya, B. A. (2006). Evaluating scale functions of spectrally negative Lévy processes. Preprint. 